| Original | Article |

\title{
Effects of intra-articular autologous mesenchymal stem cell injection under ultrasonogram guidance in patients with osteoarthritis knee
}

\author{
Md. Nuruzzaman Khandaker, Md. Ashraful Haque, Md. Ali Emran and Fatema Newaz
}

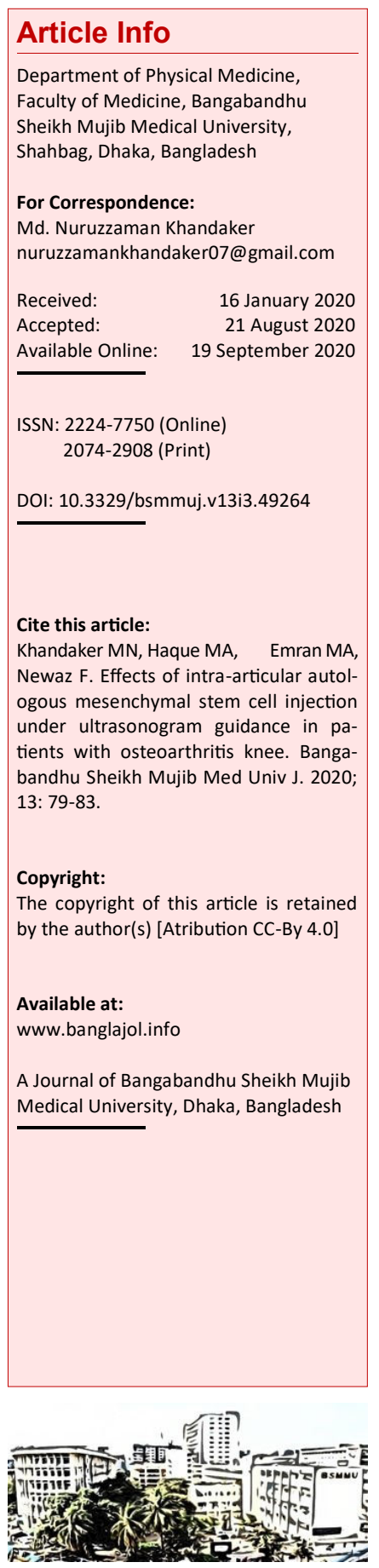

\section{Abstract}

Mesenchymal stem cells therapy opened new perspective in the management of osteoarthritis. This study focuses on a randomized controlled trial pilot on 10 patients of osteoarthritis knees to evaluate the intra-articular injections of mesenchymal stem cells could improve the symptoms, functional limitation and regeneration of cartilage. Mesenchymal stem cells were obtained from peripheral blood after mobilization of blood cell with granulocyte colony stimulating factor by apheresis machine. Single dose of mesenchymal stem cells were injected into the affected joints, WOMAC questionnaires scale used to record pre and post-treatment improvement, cartilage thickness measured by high frequency ultrasonogram. Four patients were having Grade II and six were having Grade III osteoarthritis. Kellgren-Lawrence scale was used for radiological grading. Pain score were decreased at the end of 24th weeks from $14.8 \pm 2.2$ to $4.1 \pm 1.3$, Stiffness score decreased from $5.8 \pm 0.6$ to $0.7 \pm 0.6$, Physical function limitation score reduced from $46.7 \pm 6.5$ to $20.3 \pm 2.5$. There was minor improvement of range of motion and cartilage thickness changes. This study demonstrates that mesenchymal stem cells injections improves all parameters of knee osteoarthritis and minimal regeneration of damaged cartilage.

\section{Introduction}

Osteoarthritis is a degenerative process of the articular cartilage, involving the immune system, and producing local inflammatory reactions with production of pro-inflammatory cytokines and metalloproteinases. The knee joint is frequently affected in Asian countries. $\underline{1,2}$ The prevalence increases with age, women are suffering more than men. 3 It is the cause of disability among the elderly population. $\underline{4}$ It is an important financial burden for the global economy. $\cdot 5$

Osteoarthritis of knees clinically manifested by joint pain, gelling stiffening, joint instability, difficulty to walking, jogging, squatting and stair climbing. During physical examination, mainstream finding are joint line tenderness, crepitus on movement, limitation of range of movement, joint effusion and variable levels of inflammation. A good number of patient present with quadriceps muscles wasting, difficulty to stand from sitting leading to physical disability, loss of functional capacity, reduces quality of life and increases the risk of other co-morbidity. 6 The recent report of World Health Organization indicates that osteoarthritis knee become the 4th most important cause of disability in women, and the 8th most important cause of disability in men. 6
There are many factor responsible for cartilage degeneration such as aging process, trauma, overuse and overweight, genetic predisposition, inflammatory and autoimmune arthritis, metabolic arthritis, infectious arthritis, etc. $?$

When the healing process of injured cartilage becomes slow, then complete repair of damage cartilage is not possible, which leading to secondary fibrosis process ultimately the process of degeneration gradually continues.

Various modes of treatments have been advocated for treating osteoarthritis knee such as rest, non-steroidal anti-inflammatory drugs, night splints, keeping appropriate wedge in sole, soft heel pad or well-padded medial arch support, stretching and strengthening exercises of quadriceps muscle, joint protective measure, application of different physical modalities and Intra-articular corticosteroid injection. Unfortunately, no curative treatment is available yet to reverse the process. The recurrence rate is common in most of the conservative measurement. There are also numerous surgical procedures available to manage osteoarthritis knee, that includes knee arthoplasty, osteotomy, micro fracture or micro drilling surgery, autologous chondrocyte implantation, mosaic plasty, and matrix-guided autologous chondrocyte implantation, 9 Appropriate surgical mana- 
gement of osteoarthritis is determined by specific patient symptoms, clinical and radiographic findings, circumstances, and expectations. While younger patients with isolated single compartmental osteoarthritis may benefit from a high tibial osteotomy or compartmental knee arthroplasty, patients with advanced and multiple compartmental osteoarthritis are more likely to benefit from total knee arthroplasty. The routine use of arthroscopic debridement for the treatment of osteoarthritis has been challenged recently by several randomized control studies demonstrating no significant difference between arthroscopic management and placebo in the treatment of knee osteoarthritis. $\underline{10,11}$

Although many non-surgical and surgical treatment modalities improve pain and function in osteoarthritis of knees, none can alter the natural history of the disease process. Recently, there has been increased focus on the potential role of stem cells in the management of osteoarthritis. Mesenchymal stem cells are non-hematopoietic cells which can be easily isolated from bone marrow and other tissues, such as adipose, umbilical cord, and peripheral blood. The mesenchymal stem cells have a multipotent capacity to differentiate into a variety of other cell types, including osteoblasts, adipocytes, chondrocytes, myoblasts, and neurons. 12,13 In response to stimuli, mesenchymal stem cells have the ability of homing to the target tissue. Mesenchymal stem cells also have the immunosuppressive and anti-inflammatory properties. They do not express major histocompatibility complex-II (MHC-II), CD80, CD86, and CD40, and minimally express MHC-I on the cell surface.12, 14 These characteristics have made mesenchymal stem cells a promising cell source for tissue engineering, mesenchymal stem cells used in treating degeneration muscle and a tendon injury which were well tolerated and provide sustain pain relief, improve patient's functional impairment and effective with fewer adverse reaction. $\underline{15}$

As a potential cell-based therapy for cartilage repair, mesenchymal stem cells have been suggested for treating diseased articular cartilage because of their ability to differentiate into chondrocytes and their paracrine effects of secreted bioactive materials. $\underline{16-18}$

Currently, several clinical studies concerning the use of mesenchymal stem cells as a cell-based treatment for knee osteoarthritis have reported encouraging the outcomes after mesenchymal stem cell-based treatment for knee osteoarthritis.19-23 However to date, there is no study carried out to observe the effect of peripheral blood mesenchymal stem cells in the management of osteoarthritis knee as cartilage regeneration. Therefore, this study of single dose intra-articular injections of autologous peripheral blood mesenchymal stem cells in knee joints without prior surgery carried out whether this noninvasive procedure prevent joint destruction and regenerate the articular cartilage osteoarthritis knee.

\section{Materials and Methods}

This randomized controlled trial pilot study was conducted on 10 patients of osteoarthritis knees from July to December, 2018. No control group was selected for comparison. Enrolment of patients with the following clinical features, irrespective of sexes, aged over 45 years. Knee osteoarthritis was confirmed by clinical, radiological evaluation, with unilateral affection and disease duration at least 6 months. They had moderate to severe bilateral osteoarthritis knee, usual complain were mechanical pain of knees, aggravated with walking or climbing stairs, gelling pain, crepitus, restricted range of motion, and limitation of joint motion. Xray of knee joints both view anterior posterior and lateral view in standing position were obtained from each participant at the beginning of the study for osteoarthritis grading, Kellgren and Lawrence radiological classification system used for grading of osteoarthritis knee. Among them, six patients were in Grades II and four patients were in Grade III.

The following parameters were checked before mesenchymal stem cells injection and at subsequent follow-up: Pain on visual analogue scale, walking distance and appearance of pain, climbing number of stairs that produce pain, the resting time to induce the gelling pain, the range of motion, and the presence of synovial fluid. Initial visual analogue scale, which evaluated pain on a numerical rating scale from 0 to 10 , where 0 signified no pain and 10 signified the worst pain experienced ever by the patient, and The Western Ontario and McMaster Universities Osteoarthritis Index (WOMAC) scores also recorded. Patients with systemic arthritis, knee infection or surgery in the last 6 months, an intra-articular injection in the past 3 months, malignant or traumatic lesions (joint fracture, meniscal or ligament injury) were excluded from the study. Visual analogue scale and WOMAC score were recorded at baseline, 6th week, $12^{\text {th }}$ week, and $24^{\text {th }}$ week of intervention.

\section{Mesenchymal stem cell collection procedure}

A proper work-up was performed, before starting mesenchymal stem cells collection including medical history, physical examination, and complete blood count and biochemical profile. Infectious diseases such as hepatitis B and C were also ruled out. Stimulation and harvesting of mesenchymal stem cells after overall assessment, autologous mesenchymal stem cells stimulation was started with the subcutaneous administration 
of granulocyte colony stimulating factor in a dose of $5-10 \mu \mathrm{g} / \mathrm{kg} /$ day for $5 / 6$ days on an outpatient basis (Mollison's Blood Transfusion in Clinical Medicine). A complete blood count was performed to determine the increase in cell concentration in peripheral blood on the day of mesenchymal stem cells harvesting (minimum white blood cell count should be more than $5000 / \mu \mathrm{L}$ from baseline). After harvesting, mesenchymal stem cells were collected by apheresis machine (Fresenius Kabi, Germany) in a desired volume. Mesenchymal stem cells $(3 \mathrm{~mL})$ was sent for flocytometric examination to see the qualitative and quantitative assessment. Flowcytometry enumeration of CD34+, CD45+ cells and viability assessment were made with the single-platform ISHAGE (International Society of Hematotherapy and Graft Engineering) technique in a Bacton Dickenson FACS Verse (USA) cytometer with anti-CD34, anti-CD45 antibodies. Mesenchymal stem cells were not selected or separated by apheresis nor cultured for induction or expansion.

\section{Intra-articular cell administration}

Intra-articular mesenchymal stem cell injection given on the same setting. The patient was placed in a supine position with the affected knee flexed to 30 degree. After taking proper aseptic precaution $2 \mathrm{~mL}$ of $1 \%$ lidocaine given at the puncture site, $8 \mathrm{~mL}$ of concentrate peripheral blood derived Mesenchymal stem cells was injected with an 20G 1" needle through infra-patellar immediately lateral to the patellar tendon into the lateral compartment of intra -articular space in the affected knee under the guidance of high frequency musculoskeletal ultrasonogram, All patients were injected by the same investigator.

Mesenchymal stem cells collection and intraarticular injection were given on an outpatient basis. Proper counseling regarding activities of daily livings, joint protection measure and post injection care given to all patients. Relative rest for 3 days advised after injection of stem cell. Range of motion exercise and isometric form of quadriceps muscle strengthening exercise were initiated after 3 days of relative rest. At the end of $2^{\text {nd }}$ week of post injection, patients were participated stretching and resisted

\section{Table I}

\begin{tabular}{|lrrr|}
\hline Variable & Pretreatment & Post treatment & p value \\
\hline Pain & $14.8 \pm 2.2$ & $4.1 \pm 1.3$ & $<0.001$ \\
Stiffness & $5.8 \pm 0.6$ & $0.7 \pm 0.6$ & $<0.001$ \\
Limitation of physical function & $46.8 \pm 6.5$ & $20.3 \pm 2.6$ & $<0.001$ \\
Total WOMAC Score & $64.9 \pm 10.2$ & $24.9 \pm 2.8$ & $<0.001$ \\
Data are mean \pm SD & & & \\
\hline
\end{tabular}

strengthening exercise of quadriceps muscle. Any anti-inflammatory drug or immunosuppressive drug was not prescribed after the procedure. At the same time we informed the patients in respect of joint protective measure, proper instruction given in relating to daily living activities and to wear well cushioned shoe.

\section{Results}

Table I shows the comparison of WOMAC scores between pre- and post-treatment ( 24 weeks). There was statistically significant difference between preand post-treatment, as the $\mathrm{p}$ value was $<0.05$ (obtained by Student's t-test). WOMAC sub-scale scores of the patients at the beginning of treatment, the mean pain score were $14.8 \pm 2.2$ significantly reduced to $4.1 \pm 1.3$ at $24^{\text {th }}$ week. Other components of WOMAC index, stiffness and physical function limitation also decreased remarkably from (5.8 \pm $0.6)$ to $(0.7 \pm 0.6),(46.8 \pm 6.5)$ to $(20.3 \pm 2.6)$. Total WOMAC index score initially was $(64.9 \pm 10.2)$, then it became $(24.9 \pm 2.8)$ at $24^{\text {th }}$ weeks. These indicated that there were remarkable changes after intraarticular mesenchymal stem cells injection in knee joints.

Others physical parameters range of motion and articular cartilage thickness were not improve in significant. There was no radiological improvement, but ultrasonological changes of articular cartilage thickness showed mild improved in few cases.

\section{Discussion}

In this study of mesenchymal stem cells injection for the treatment of osteoarthritis shows that intraarticular injection of mesenchymal stem cells is a safe procedure without any complication. This study results here are preliminary reports at $24^{\text {th }}$ week only. This study results revealed that after injection of mesenchymal stem cells in osteoarthritis knee are inspiring, but not outstanding.

The positive subjective parameters improvement can be explained in animal studies where one of the main effects of mesenchymal stem cells transplantation in the joint is the suppression of the synovial inflammation that reduce pain, stiffness and improve functional limitation. $\underline{\underline{4}}$ Cartilage thickness changes explained the transplanted mesenchymal stem cells into the joints generate a microenvironment to repair the cartilage. 25 This repair may be due to the effect of transforming growth factor $\beta$ on local mesenchymal stem cells and chondrocyte, switching the Smad1/5/8 phosphorylation (due to the presence of A Disintegrin and Metalloproteinase with Thrombospondin Motifs [AD-AMTS5], probably because of synovial inflammation) to 
Smad2/3 phosphorylation which is key protector of joint normal cartilage. $\frac{26-29}{}$ However, the mild improvement of objective signs cannot be explained by the placebo effect, further randomized clinical trial will clarify this matter. The global improvement of subjective parameters was for both knees, also suggesting a possible central role of mesenchymal stem cells. The results obtained by Centeno et al (2008) $\underline{30}$ on their unique case were much better than the present results, perhaps due to their procedure (injection of platelets and hematopoietic stem cells as well as mesenchymal stem cells). However, the growth of mesenchymal stem cell in culture shows fibroblast-like cells. As Centeno et al. report, we should be sure whether this is a fibroblast-like cells or true growth of cartilage. The main problem is to find the required number of cells for injection, in order to have the optimal response. Whether one injection will be enough, or more than one in a time period to reach the desired result, is another question to be answered in future works.

\section{Conclusion}

Mesenchymal stem cells injection into the knee joint could improve the patient's symptoms.

\section{Financial Support}

Self-funded

\section{Ethical Issue}

The research carried out here with human subjects was in compliance with the Helsinki Declaration. It was approved by the Institutional Review Board of Bangabandhu Sheikh Mujib Medical University (BSMMU/2018/1590). A well-informed, voluntary signed written informed consent was obtained from all patients before enrollment of this clinical trial, each patient enjoyed every right to participate or refuse or withdraw from the study at any point in time.

\section{Conflict of Interest}

The authors declare no conflict of interest.

\section{Acknowledgement}

An author acknowledges the support from the Department of Transfusion Medicine and Department of Microbiology, Bangabandhu Sheikh Mujib Medical University.

\section{References}

1. Davatchi F. Rheumatic diseases in the APLAR region. APLAR J Rheumatol. 2006; 9: 5-10.

2. Elders MJ. The increasing impact of arthritis on public health. J Rheumatol (Suppl). 2006; 60: 6-8.

3. Felson DT, Naimark A, Anderson J. Kazis L, Castelli W, Meenan RF. The prevalence of knee osteoarthritis in the elderly: The Framingham osteoarthritis. study. Arthritis Rheum. 1987; 30: 914 -18 .

4. Shakoor MA.Taslim MA, Ahmed MS, Hasan SA Clinical profile of patients with osteoarthritis of the knee: A study of 162 cases. IJPMR. 2009; 20: 44-47.

5. Neogi T. The epidemiology and impact of pain in osteoarthritis. Osteoarthritis Cartilage. 2013; 21: 1145-53.

6. Shipley M, Rahman A, Gradaigh D, Compston JE. Rheumatology and bone disease. In: Kumar and Clark's Clinical medicine. Kumar P, Clark M (eds). $7^{\text {th }}$ ed. Edinburg, Elseiver Saunders, 2009, 499-570.

7. Dahaghin S, Tehrani-Banihashemi A, Faezi ST, et al. Squatting, sitting on the floor, or cycling: Are lifelong daily activities risk factors for clinical knee osteoarthritis Stage III results of a communitybased study. Arthritis Rhematol. 2009; 61: 1337-42.

8. Haq SA, Davatchi F, Dahaghin S, et al. Development of a questionnaire for identification of the risk factors for osteoarthritis of the knees in developing countries: A pilot study in Iran and Bangladesh. An ILAR-COPCORD phase III study. Int J Rheum Dis. 2010; 13: 203-14.

9. Murray CJL, Lopez AD. The global burden of disease. Geneva, World Health Organization, 1997.

10. Kirkley A, Birmingham TB, Litchfield RB, et al. A randomized trial of arthroscopic surgery for osteoarthritis of the knee. N Engl J Med. 2008; 359: 1097-107.

11. Moseley JB, O’Malley K, Petersen NJ, et al. A controlled trial of arthroscopic surgery for osteoarthritis of the knee. N Engl J Med. 2002; 347: 81-88.

12. Pittenger MF, Mackay AM, Beck SC, Jaiswal RK, Douglas R, Mosca JD, et al. Multilineage potential of adult human mesenchymal stem cells. Science 1999; 284: 143-47.

13. Jiang $\mathrm{YH}$, Jahagirdar BN, Reinhardt RL, Schwartz $\mathrm{RE}$, Keene CD, Ortiz-Gonzalez XR, et al. Pluripotency of mesenchymal stem cells derived from adult marrow. Nature 2002; 418: 41-49.

14. Tse WT, Beyer W, Pendleton JD, D'Andrea A, Guinan EC. Bone marrow derived mesenchymal stem cells suppress $\mathrm{T}$ cell activation without inducing allogeneic anergy. Blood 2000; 96: 241a.

15. Woodbury D, Schwarz EJ, Prockop DJ, Black IB. Adult rat and human bone marrow stromal cells 
differentiation into neurons. J Neurosci Res. 2000; 61: 364-70.

16. Barry F, Murphy M. Mesenchymal stem cells in joint disease and repair. Nat Rev Rheumatol. 2013; 9: 584-94.

17. Caplan AI, Dennis JE. Mesenchymal stem cells as trophic mediators. J Cell Biochem. 2006; 98: 107684.

18. Oreffo RO, Cooper C, Mason C, Clements M. Mesenchymal stem cells: Lineage, plasticity, and skeletal therapeutic potential. Stem Cell Rev. 2005; 1: 169-78.

19. Jo $\mathrm{CH}$, Lee $\mathrm{YG}$, Shin $\mathrm{WH}$, et al. Intra-articular injection of mesenchymal stem cells for the treatment of osteoarthritis of the knee: A proof of concept clinical trial. Stem Cells. 2014; 32: 1254-66.

20. Kim YS, Choi YJ, Suh DS, et al. Mesenchymal stem cell implantation in osteoarthritic knees: Is fibrin glue effective as a scaffold? Am J Sports Med. 2015; 42: $176-85$.

21. Koh YG, Choi YJ, Kwon OR, Kim YS. Second-look arthroscopic evaluation of cartilage lesions after mesenchymal stem cell implantation in osteoarthritic knees. Am J Sports Med. 2014; 42: 1628-37.

22. Koh YG, Jo SB, Kwon OR, et al. Mesenchymal stem cell injections improve symptoms of knee osteoarthritis. Arthroscopy 2013; 29: 748-55.

23. Orozco L, Munar A, Soler R, et al. Treatment of knee osteoarthritis with autologous mesenchymal stem cells: Two-year follow-up results. Transplantation 2014; 97: e66-e68.

24. Schulze-Tanzil G. Activation and dedifferentiation of chondrocytes: Implications in cartilage injury and repair. Ann Anat. 2009; 191: 325-38.

25. Van Lent PL, van den Berg WB. Mesenchymal stem cell therapy in osteoarthritis: Advanced tissue repair or intervention with smouldering synovial activation? Arthritis Res Ther. 2013; 15: 112.

26. Roelofs AJ, Rocke JP, De Bari C. Cell-based approaches to joint surface repair: A research perspective. Osteoarthritis Cartilage. 2013; 21: 892-900.

27. Glasson SS, Askew R, Sheppard B et al. Deletion of active ADAMTS5 prevents cartilage degradation in a murine model of osteoarthritis. Nature 2005; 434 $644-48$.

28. Li J, Anemaet W, Diaz MA et al. () Knockout of ADAMTS5 does not eliminate cartilage aggrecanase activity but abrogates joint fibrosis and promotes cartilage aggrecan deposition in murine osteoarthritis models. J Orthop Res. 2011; 29: 51622.

29. Van der Kraan PM, Goumans ML. Age-dependent alteration of TGF-b signalling in osteoarthritis. Cell Tissue Res. 2012; 347: 257-65.

30. Centeno CJ, Busse D, Kisiday J, Keohan C, Freeman $\mathrm{M}$, Karli D. Increased knee cartilage volume in degenerative joint disease using percutaneously implanted, autologous mesenchymal stem cells. Pain Physician. 2008; 11: 343-53. 